\title{
TRANSFER FUNCTION ANALYSIS OF ULTRASONIC TIME-INTENSITY MEASUREMENTS
}

\author{
PAI-CHI Li* ${ }^{* \dagger}$ and MEI-JU YANG* \\ *Department of Electrical Engineering, National Taiwan University, Taipei, Taiwan; and ${ }^{\dagger}$ Division of Medical \\ Engineering, National Health Research Institutes, Taipei, Taiwan
}

(Received 7 January 2003; revised 7 April 2003; in final form 15 April 2003)

\begin{abstract}
Time-intensity measurements of ultrasonic-contrast microbubbles based on the dilution theory have been used to assist blood flow estimation. The compartment model has been employed to describe the dilution process. Under the linear and time-invariant assumption, the time-intensity curve measured at the output of a compartment (i.e., blood mixing chamber) is the convolution of the input time-intensity curve with the compartment's transfer function. Thus, transfer function analysis is possible using deconvolution when the temporal variations in both the input and the output intensities are available. Note that the linear and time-invariant assumption requires a constant flow rate because, with flow pulsation, the flow rate changes with time and the mixing process becomes time varying. Thus, the purpose of this paper was to study the effects of flow pulsation on time-intensity measurements. In addition, a deconvolution technique based on a recursive least squares approach is used for transfer function analysis. Both simulations and experiments were performed; the results from which indicate that the pulsation generally does not affect the validity of time-intensity-based flow estimation. The proposed deconvolution technique is also effective for both constant and pulsatile flows; thus, permitting transfer function analysis in various flow conditions. One potential application of this transfer function analysis is to remove the effects of a noninstantaneous input function. The results from this paper lead to future work in brain-perfusion estimation based on extracranial time-intensity measurements. (E-mail: paichi@cc.ee.ntu.edu.tw) (c) 2003 World Federation for Ultrasound in Medicine \& Biology.
\end{abstract}

Key Words: Ultrasound, Contrast agent, Blood flow, Deconvolution, Time-varying system, Transfer function.

\section{INTRODUCTION}

In medical ultrasonic imaging, microbubble-based contrast agents have been used to assist blood flow estimation by increasing the acoustic backscattered intensity of the blood (Kemper et al. 1985; Cheirif et al. 1989; Kaul et al. 1989; Ophir and Parker 1989; Shung et al.; Porter et al. 1993; Schwarz et al. 1993a, 1993b, 1996; Wilson et al. 1993; de Jong 1996; Goldberg 1997; Yeh et al. 2001; Li et al. 2002; Seidel et al. 2002). New imaging methods have also been developed that utilize the nonlinear response of the microbubbles, particularly when the impinging sound wave is near the bubble's resonance frequency (Gramiak et al. 1969; Burns 1996; Krishnan et al. 1998; Morgan et al. 1998; Haider and Chiao 1999; Shi et al. 1999; Hoff 2000; Kirkhorn et al. 2001; Phillips 2001). One example of a contrast-agent-assisted flow estimation method is the time-intensity-based technique (Schwarz et

Address correspondence to: Dr. Pai-Chi Li, Department of Electrical Engineering, National Taiwan University, No. 1, Sec. 4, Roosevelt Road, Taipei 106 Taiwan. E-mail: paichi@cc.ee.ntu.edu.tw al. 1993a, 1993b, 1996; Yeh et al. 2001; Li et al. 2002). This is based on the dilution of microbubbles in blood, with the bubble concentration being monitored by measuring the temporal changes in backscattered intensity. A linear relationship is typically required between the measured acoustic intensity and the microbubble concentration. The measured temporal changes in intensity are also known as the time-intensity curve (TIC). Several parameters derived from the TIC have been correlated with the flow rate. Li et al. (2002) showed that the change in the microbubble washout rate is linearly proportional to the actual volumetric flow rate. That study also showed that the peak intensity of the TIC is approximately linearly proportional to the volume concentration of the injected contrast agent under carefully controlled imaging conditions. Thus, the possibility for TIC-based quantitative perfusion analysis has been demonstrated.

The compartment model has been adopted to interpret the time-intensity data in medical imaging (Gobbel and Fike 1994; Chen et al. 1998). In this case, the blood pool in the human body is considered to consist of 
multiple blood-mixing compartments. The indicator-dilution theory provides a mathematical basis for characterizing the blood-mixing process. In addition, the measured TIC represents a convolution of the input TIC and the dilution transfer function in the region-of-interest (ROI). Thus, flow parameters at various circulation stages can be related to one another (Chen et al. 1998). Note that only the dilution transfer function is of interest for flow estimation and, so, the influence of the input function should be removed. One way to remove this influence is using deconvolution (Gobbel and Fike 1994). In fluorometry, for example, a multicompartment model for fluorescein dye distribution has been employed to describe the relationship among perfusion, dye kinetics and fluorescence readings (Ostrander et al. 1990). Each compartment can be further modeled as a linear and time-invariant (LTI) system. The Wiener filter has been applied to deconvolve the measured data to determine the transfer function between the circulation system and the indicator (Bates 1991; Gobbel and Fike 1994). The blood flow characteristics can be evaluated from this transfer function.

In ultrasound (US), the linearity of the relationship between the measured acoustic intensity and the bubble concentration has been investigated experimentally (Schwarz et al. 1993b; Wilson et al. 1993; Yeh et al. 2001); the compartment model has also been adopted (Schwarz et al. 1993a; Chen et al. 1998; Yeh et al. 2001; Li et al. 2002). It was found that the measured acoustic intensity is generally a nonlinear function of the concentration. Nonetheless, relative flow assessment is still possible if the concentration of the microbubbles is within a defined range (Yeh et al. 2001).

The compartment model has been widely used, but deconvolution techniques have only been applied to imaging modalities other than ultrasound. More accurate diagnostic information would be available from US investigations if proper deconvolution techniques could be developed for estimating the transfer function. For example, it is possible to remove the effects of the microbubble concentration at the input end of the ROI when the injection of microbubbles is not instantaneous, such as when the injection is not a bolus or when the ROI is far away from the injection site. Another example is to estimate the transfer function in a perfused area where the acoustic access may be limited, a potential clinical example of which is the measurement of brain perfusion (Yeh et al. 2003).

Note that deconvolution techniques are generally developed based on the LTI assumption. In many clinical settings, however, the transfer function is time varying because the flow rate changes with time. Thus, the applicability of the deconvolution techniques needs to be tested. To this end, it was the main purpose of this study to address the following two issues. First, the effects of time-varying flows on the measured TIC were investigated. The hypothesis that such effects can be ignored when using the TIC-based flow-estimation methods was also tested. Second, an effective deconvolution technique was developed. Specifically, a recursive least squares (RLS) method is proposed and its performance was investigated on both constant flows and pulsatile flows. Addressing these two issues allows determination of the feasibility of transfer function analysis in US imaging. Note that, although the compartment model has been adopted for various organs, such as the heart (DiBella et al. 1997; Nouri-Baranger et al. 2001), the liver (Heikkonen and Mantyla 1991) and the brain (Meikle et al. 1998; Zhou et al. 1997), brain-perfusion estimation is of particular interest in this paper because the extracranial measurements potentially can alleviate the limited acoustic access of the brain (Schoning and Scheel 1996; Seidel et al. 2002).

This paper is organized as follows. A mathematical model for time-varying systems based on the principle of superposition is first developed and verified. The RLS deconvolution technique is then described and tested. Finally, the paper concludes with a discussion on potential clinical applications of the proposed technique.

\section{MODELING A TIME-VARYING SYSTEM}

Review of the input-output relationship for an LTI system

Assuming a constant flow, the mixing of the contrast agent can be modeled as an LTI process (Chen et al. 1998; Yeh et al. 2001; Li et al. 2002). In this case, the time-intensity analysis is based on the dilution theory and the assumption that the backscattered acoustic intensity is linearly proportional to the microbubble concentration. The echo intensity measured at the output of a mixing chamber, denoted by $I_{\mathrm{o}}(t)$, can be expressed as:

$$
I_{\mathrm{O}}(t)=\int_{-\infty}^{\infty} h(\xi) I_{\mathrm{I}}(t-\xi) d \xi
$$

where $h(\xi)$ is the transfer function of the mixing chamber and $I_{\mathrm{I}}(t)$ is the input function. Based on the dilution theory, and assuming instant and complete mixing in the compartment, the transfer function contains flow information and can be represented by:

$$
h(t)= \begin{cases}0 & t<0 \\ Q / V \cdot e^{-Q t / V} & t \geq 0\end{cases}
$$

where $Q$ represents the constant volumetric flow rate and $V$ is the compartment's effective mixing volume. Thus, blood flow estimation can be performed if the transfer 
function is available. In practice, an overall delay accounting for the time needed for the microbubbles to travel through the compartment may also be added to the above equation.

The TIC can also be used to derive the mean transit time (MTT), defined as:

$$
M T T=\frac{\int_{0}^{\infty} t I(t) d t}{\int_{0}^{\infty} I(t) d t} .
$$

The MTT represents the time for the entire fluid volume to pass through the mixing volume. With a bolus injection (i.e., an approximately instantaneous injection), the output TIC is close to the transfer function. In this case, we have:

$$
M T T=\frac{V}{Q} .
$$

Note that the term $V / Q$ is also known as the washout time constant, and its inverse is the washout rate. When the input is not a bolus, it can also be shown that the MTT of the output function ( $\left.M T T_{\text {out }}\right)$ equals the sum of the $M T T$ of the input function $\left(M T T_{\mathrm{in}}\right)$ and the $M T T$ of the compartment transfer function ( $\left.M T T_{\mathrm{com}}\right)$ (Yeh et al. 2001):

$$
\mathrm{MTT}_{\text {out }}=\mathrm{MTT}_{\text {in }}+\mathrm{MTT}_{\text {com }} .
$$

\section{Input-output relationship of a time-varying system}

The transfer function described in eqn (2) is a function of the flow rate $Q$. Thus, it becomes time varying if the flow rate is time varying. A time-varying system can be characterized by a 2-D transfer function $h(t, \xi)$, which depends on both the excitation time $\xi$ and the observation instant $t$ (Claasen and Mecklenbrauker 1982). Based on the principle of superposition, the output $I_{\mathrm{o}}(t)$ can be expressed as:

$$
I_{\mathrm{O}}(t)=\int_{-\infty}^{\infty} h(t, \xi) I_{I}(\xi) d \xi .
$$

If the system is LTI, $h(t, \xi)$ depends only on the time difference $t-\xi$, in which case eqn (6) reduces to a formulation similar to eqn (1). On the other hand, eqn (6) can be modified so that it resembles a convolution integral by introducing a new function:

$$
p(t, \xi)=h(t, t-\xi) .
$$

Then,

$$
I_{\mathrm{O}}(t)=\int_{-\infty}^{\infty} p(t, \xi) I_{\mathrm{I}}(t-\xi) d \xi
$$

Both $h(t, \xi)$ and $p(t, \xi)$ are referred to in the literature as the transfer function for a linear time-varying system. The function $h(t, \xi)$ gives the response at time $t$ for an impulse excitation at time $\xi$. The function $p(t, \xi)$, on the other hand, represents the response at time $t$ caused by an impulse input that is applied $\xi$ earlier in time. The transfer function for a causal, linear, time-varying system is zero for $t<\xi$. Hence,

$$
h(t, \xi)=0, t<\xi
$$

or, equivalently

$$
p(t, \xi)=0, \xi<0 .
$$

Finally, the time-varying transfer function $h(t, \xi)$ is given by:

$$
h(t, \xi)=\left\{\begin{array}{ll}
0 & t<\xi \\
Q(\xi) / V \cdot e^{-Q(\xi) t / V} & t \geq \xi
\end{array},\right.
$$

where $Q(\xi)$ represents the time-varying flow rate. Note that the MTT of the TIC can still be defined as in eqn (3) in a time-varying system. In addition, it will be demonstrated that the MTT of a time-varying flow is inversely proportional to the mean flow rate.

\section{EFFECTS OF FLOW PULSATION ON TIC MEASUREMENTS}

\section{Flow model}

The model shown in Fig. 1 was used for both simulations and in vitro experiments (Yeh et al. 2001; Li et al. 2002). The model consisted of two sphericalcompartment phantoms in cascade. For in vitro measurements, pulsatile and constant flows were emulated using a commercially available flow system (UHDC flow system, Shelley Medical Imaging Technologies, North York, Ontario, Canada). A blood-mimicking fluid was also used (Shelly Medical Imaging Technologies). As shown in this figure, the input of chamber $\mathrm{B}$ was the output of chamber A. The purpose of compartment A was to simulate prolonging of the input function (Yeh et al. 2001). The spherical-compartment phantom comprised an acrylic hollow sphere. Two silicon tubes connected to the sphere were fixed in parallel, with opposite flow directions. The inner diameter of the tubes was 5 $\mathrm{mm}$. A linear-array transducer (546L, GE Medical Sys- 


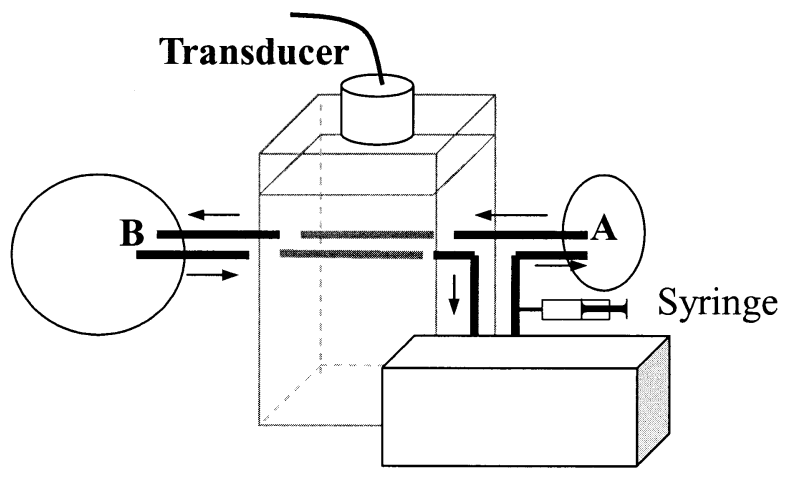

Fig. 1. Flow model for both simulations and in vitro experiments: two compartment phantoms in cascade.

tems, Milwaukee, WI) was placed $2 \mathrm{~cm}$ above the tubes. The input and output TICs were simultaneously measured using a commercial US machine (LOGIQ 500 System, GE Medical Systems). The mechanical index was 0.4 to avoid significant bubble destruction. A commercial contrast agent (Levovist ${ }^{\circledR}$, Schering, Berlin, Germany) was injected at the input side of chamber $A$. Concentration of the contrast agent was $260 \mathrm{mg} / \mathrm{mL}$; a bolus of $4 \mathrm{~mL}$ was manually injected into the system through a syringe. The display dynamic range was $48 \mathrm{~dB}$ and the time gain compensation was adjusted for image brightness uniformity. After the time gain compensation was adjusted, the settings were fixed for all time-intensity measurements. The transmit focus was at the same depth as the tubes (i.e., $2 \mathrm{~cm}$ ).

\section{Time-intensity measurements}

Grey-scale images of the transverse view of the input and the output tubes were acquired at a rate of 1 frame/s. A total of 500 image frames were acquired and digitized by a frame grabber (UPG401B, UPMOST, Taipei, Taiwan). Fundamental B-mode imaging at $5 \mathrm{MHz}$ was used. Off-line analysis was performed on a personal computer. In each frame, the mean intensity of the pixels inside the ROI (i.e., the input vessel or the output vessel) was used as the time intensity at that particular instant. The measured time-intensity curve was normalized so that the total area-under-the-curve (AUC) became unity. Antilog was performed off-line before the time-intensity curves on a linear scale were calculated. To reduce the influence of measurement noise, the time-intensity data were fitted to the following gamma function:

$$
g(t)=\alpha\left(t-t_{0}\right)^{\gamma} e^{-\beta\left(t-t_{0}\right)},
$$

where $\alpha$ and $\beta$ are scaling factors and $\gamma$ represents the skewness.

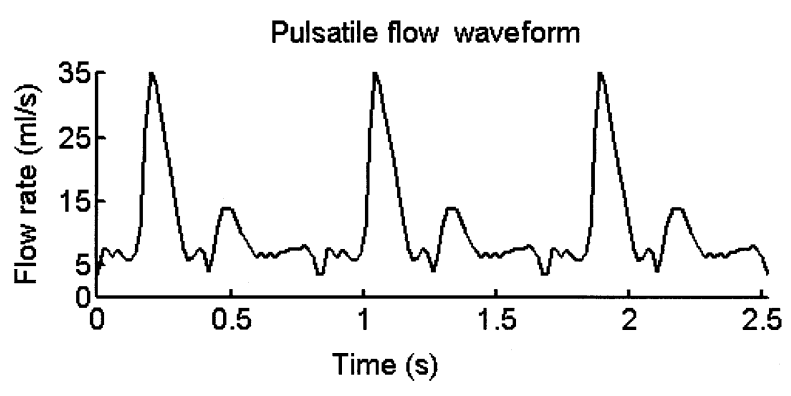

Fig. 2. An example of a pulsatile flow waveform.

Relationship between the flow rate and the MTT: Simulations

The time-varying flows used in this study were emulated carotid arterial waveforms downloaded from the UHDC flow system. The basic waveform is shown in Fig. 2, and it was scaled for five different peak flow rates: 15, 20, 25, 30 and $35 \mathrm{~mL} / \mathrm{s}$. The corresponding mean flow rates were $4.4,5.9,7.4,8.9$ and $10.4 \mathrm{~mL} / \mathrm{s}$, respectively, and these were used for the corresponding constant-flow experiments. The volumes of mixing chambers $\mathrm{A}$ and $\mathrm{B}$ were 210 and $250 \mathrm{~mL}$, respectively.

Simulation results under the above flow conditions are shown in Fig. 3. Fig. 3a is the results from the TIC simulated at the input of chamber B, and Fig. $3 \mathrm{~b}$ is at the output. The horizontal axis represents the theoretical values obtained from eqn (4) and the vertical axis corresponds to the estimated $M T T$ values. The MTTs for the constant flow are shown by the solid lines, and the dotted lines represent results for pulsatile flows. Note that, for pulsatile flows, the corresponding mean flow rate was used to calculate the theoretical values. In all cases, the correlation coefficients $(R)$ between the estimated MTT and the best-fit lines were higher than 0.99 , indicating that using the time-intensity-based method for relative measurements is feasible for both constant and pulsatile flows. In addition, the simulation results for constant flows are very close to the theoretical values, whereas the estimated MTTs for pulsatile flows are consistently larger than those for constant flows with the same mean flow rates. In other words, the flow pulsation effectively decreases the washout rate in comparison with a constant flow with the same mean flow rate.

\section{Experimental results}

The setup shown in Fig. 1 was also used for flow experiments. The chamber sizes and the flow rates were identical to those used in the previous simulations. Each flow rate was measured independently 5 times, from which the mean and SD were obtained. Typical TICs are shown in Fig. 4. Figure $4 \mathrm{a}$ and $\mathrm{b}$ show the input TIC and the output TIC, respectively, of the mixing chamber B in 
(a)

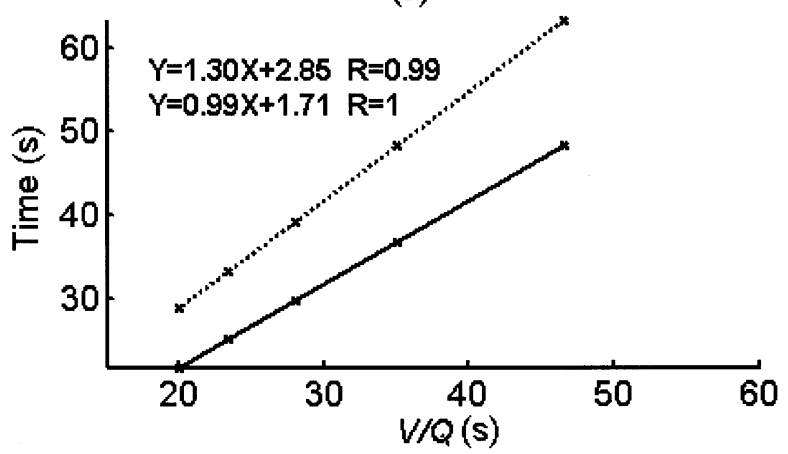

(b)

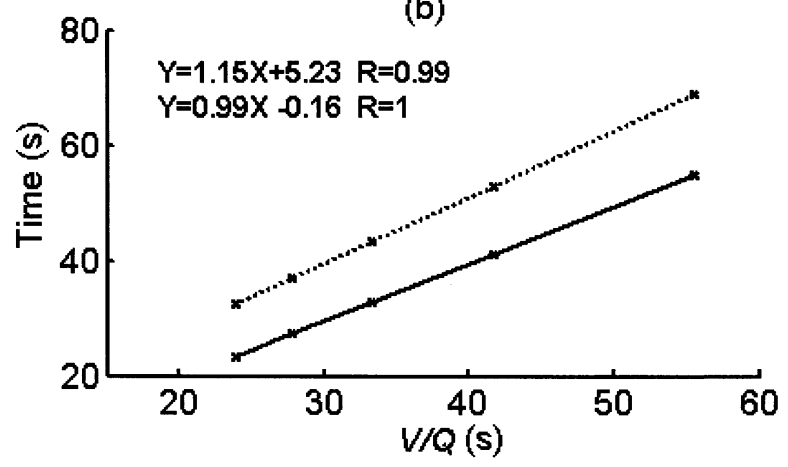

Fig. 3. Relationship between the theoretical values $(V / Q)$ and the MTT. (a) Input of mixing chamber B; (b) output of the mixing chamber. (-) constant flows; $(\cdots)$ pulsatile flows.

the pulsatile flow situation with a peak rate of $35 \mathrm{~mL} / \mathrm{s}$. Figure $4 \mathrm{c}$ and $\mathrm{d}$ show the corresponding constant-flow cases for a flow rate of $10.4 \mathrm{~mL} / \mathrm{s}$. Note that the dotted lines correspond to the original measurements and the solid lines are gamma curve fits according to eqn (12). The figure shows that the presence of pulsation does not affect the general characteristics of the measured TIC. Also note that, although the flow model had a recircu-

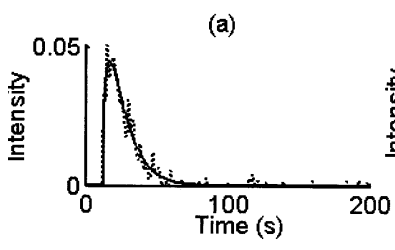

(c)
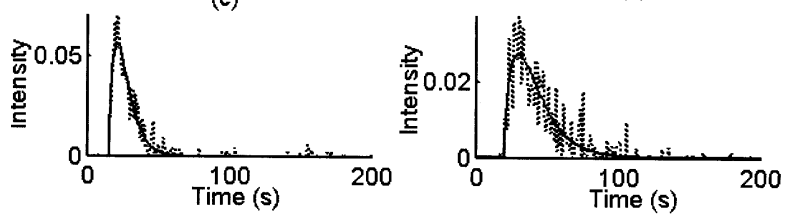

Fig. 4. Measured TIC values. (a) and (b) are for the input and the output, respectively, for a pulsatile flow with a peak rate of $35 \mathrm{~mL} / \mathrm{s}$. (c) and (d) are for a constant flow rate of $10.35 \mathrm{~mL} / \mathrm{s}$. (a)

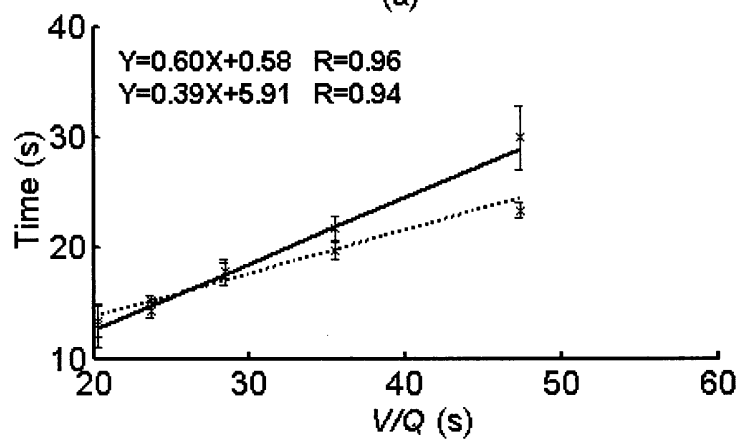

(b)

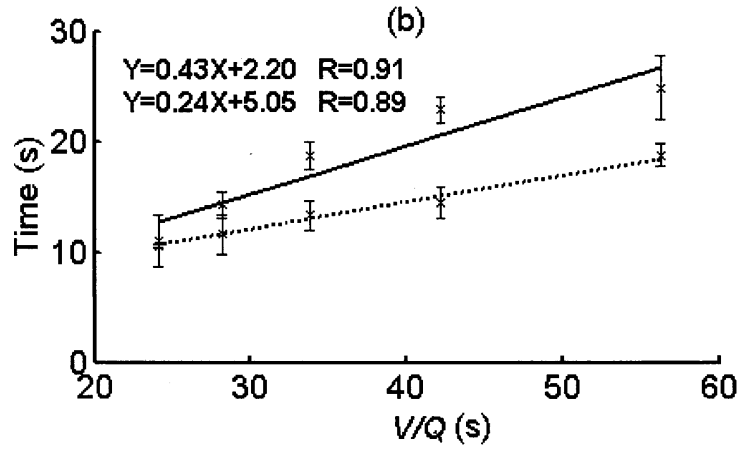

Fig. 5. The relationship between the theoretical values $(V / Q)$ and the measured MTT: (a) input, (b) output. (-) MTTs measured in constant flows; $(\cdots)$ pulsatile flow results.

lating flow, the size of the reservoir was relatively large $(3000 \mathrm{~mL})$. Thus, effects of recirculation (e.g., a very long, nearly steady-state intensity increase) were not significant.

Figure 5 plots the relationship between the theoretical (horizontal axis) and the experimentally measured MTTs (vertical axis). Figure 5a is the input of chamber B and Fig. 5b is the output. The MTTs measured in constant flows are indicated by the solid lines and those measured in pulsatile flows by the dotted lines. The measured time constants are all lower than the theoretical values obtained from the simulations. In addition, the pulsatile flow results are generally smaller than the constant-flow results. This difference is possibly attributable to the effective mixing volume being smaller than the physical volume of each mixing chamber; such a characteristic has also been reported in Li et al. (2002). Despite this discrepancy between the simulations and the experiments, the relationship between the measurement results and the theoretical values is still linear. In Fig. 5a, the two correlation coefficients between the estimated MTTs and the best-fit lines were 0.94 and 0.96 for pulsatile flows and constant flows, respectively. In Fig. 5b, the two corresponding correlation coefficients decrease slightly to 0.89 and 0.91 . Hence, the MTT can still be used for relative volumetric flow measurements. 


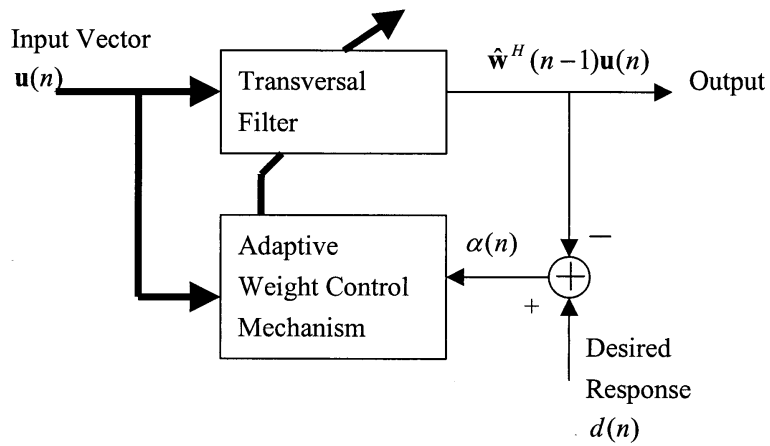

Fig. 6. Block diagram of the RLS-based deconvolution technique.

The theoretical MTT values in this study were 20 to $60 \mathrm{~s}$, all significantly larger than a heart cycle. To investigate the effects of flow pulsation on the TIC measurements when the heart cycle is close to the MTT, the pulsatile flow cycle shown in Fig. 2 was scaled from $0.846 \mathrm{~s}$ by 2 times, 5 times, 10 times and 20 times for both simulations and experiments. A peak flow rate of 20 $\mathrm{mL} / \mathrm{s}$ was used. The results show that the MTT is relatively constant under all conditions. Additional simulations were also preformed when the MTT is about the same as the heart cycle. Again, results indicated that the measurements agree well with the theory. In other words, the TIC-based method is applicable to pulsatile flows over a wide range of flow rates. Similar to previous results, the simulation results are consistently larger than the experimental results.

\section{RLS-BASED DECONVOLUTION}

The RLS adaptive filter is applied as a deconvolution technique to obtain the blood-mixing transfer function. A block diagram of the adaptive filter is shown in Fig. 6. The filter is based on the exponentially weighted least-squares method (Ljung and Soderstrom 1983; Haykin 1991). Let $\mathbf{u}(i)$ be the input TIC at a time instant $i$, where $\mathbf{u}(i)=[u(i), u(i-1), \ldots, u(i-M+1)]^{T}$ and $M$ is the number of data points used for the deconvolution, and the weighting vector $\mathbf{w}(n)=\left[w_{0}(n), w_{1}(n), \ldots, w_{M}-\right.$ $1(n)]^{T}$ is adaptively updated to minimize the error between the filter output and the desired response. At each iteration, a cost function $\gamma(n)$ is defined as:

$$
\gamma(n)=\sum_{i=1}^{a} \lambda^{n-i}|e(i)|^{2}
$$

where

$$
e(i)=d(i)-y(i)=d(i)-\mathbf{w}^{H}(n) \mathbf{u}(i),
$$

and $\lambda$ is a forgetting factor, $H$ is the Hermitian operator, and $y(i), d(i)$ and $e(i)$ represent the output, desired response and the posteriori estimation error, respectively. To minimize the cost function using the matrix-inversion lemma, the following optimal solution based on the priori estimation error $\alpha(i)$ can be found (Haykin 1991):

$$
\begin{gathered}
\hat{\mathbf{w}}(n)=\hat{\mathbf{w}}(n-1)+\mathbf{k}(n) \alpha^{*}(n) \\
\alpha(n)=d(n)-\hat{\mathbf{w}}^{H}(n-1) \mathbf{u}(n) \\
\mathbf{k}(n)=\frac{\mathbf{P}(n-1) \mathbf{u}(n)}{\lambda+\mathbf{u}^{H}(n) \mathbf{P}(n-1) \mathbf{u}(n)} \\
\mathbf{P}(n)=\lambda^{-1}\left[\mathbf{P}(n-1)-\mathbf{k}(n) \mathbf{u}^{H}(n) \mathbf{P}(n-1),\right.
\end{gathered}
$$

where * represents the complex conjugate, $\mathbf{k}(n)$ is a gain vector, and $\mathbf{P}(n)$ is the inverse of the input covariance matrix defined by the matrix-inversion lemma. In general, $\mathbf{w}(0)$ is a zero vector and $\mathbf{P}(0)$ has to be greater than $1 /[0.01 \cdot \operatorname{var}(\mathbf{u})]$ to avoid singularity. In our study, the filter order and the number of iterations are the same as the length of the input vector.

The RLS-based method is applied to both simulation and experimental data. For simulation data, results indicate that the RLS-based method is able to effectively perform deconvolution to obtain the transfer function for both constant and pulsatile flows. In addition, the estimated time constant from the RLS-based method is also in excellent agreement with the theoretical values. Nonetheless, in contrast to the results for constant flows, the pulsatile-flow results can be separated into two groups. First, the estimated washout time constants $(V / Q)$ are almost identical to the theoretical values. Second, the two MTT estimates, the MTT of the transfer function and the MTT from eqn (5), are also similar to each other. However, the two groups differ significantly, with the washout time constant $(V / Q)$ larger than the MTT. Nonetheless, the linear relationship still holds in all cases.

The performance of the RLS-based deconvolution technique using experimental data were further tested with the same experimental setup as that shown in Fig. 2. Typical measured TICs are shown by the dashed lines in Fig. 7, and the solid lines are gamma curve fits. Figure 7a shows the input and Fig. $7 \mathrm{c}$ is the output. The estimated transfer function is shown in Fig. 7b. The estimated flow rates calculated from the three curves are shown in Fig. 8 , with the ideal values indicated by the solid line with circles. The lower solid line is the MTT estimated from eqn (5) for constant flows. In all four cases, the correlation coefficients are higher than 0.89 , proving the efficacy of this approach for relative flow rate estimation from experimental data. 
(a)

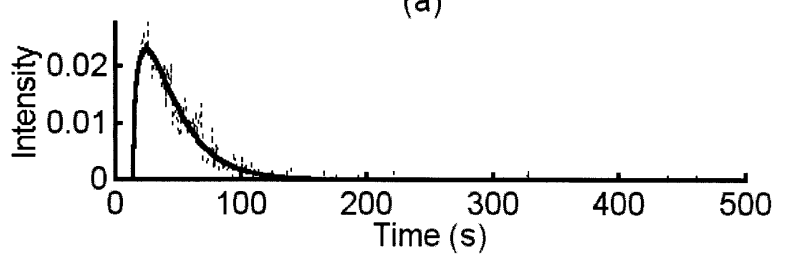

(b)

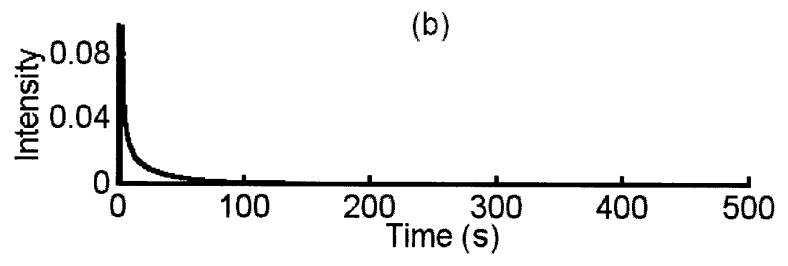

(c)

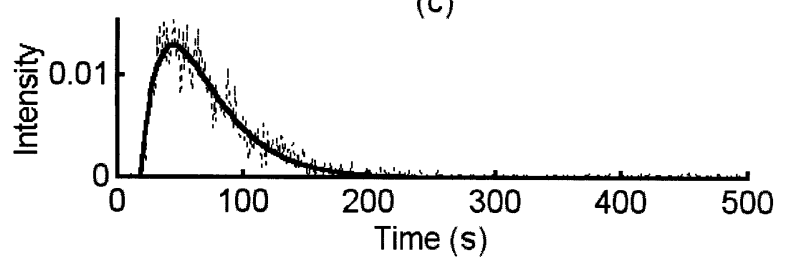

Fig. 7. TICs of a pulsatile flow: (a) input, (b) transfer function, (c) output.

\section{CONCLUSIONS}

This paper establishes a time-varying model for the TIC-based flow estimation method and proposes an RLS-based deconvolution technique. Extensive simulations were performed with this model. In addition, an in vitro experiment model was constructed. Both the simulations and the experimental results show that the RLS-

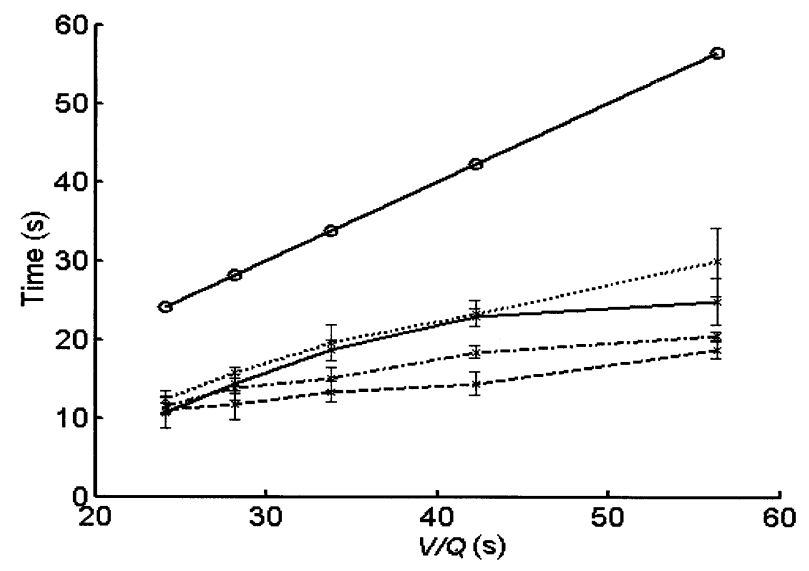

Fig. 8. Experimental results on the estimated time constants. The ideal values are indicated by the solid line with circles. (-) the MTT from the measured TIC (constant flows); (----) from the pulsatile flows, $(\cdots)$ the constant-flow results from the estimated transfer function; $(-\cdot \cdots \cdot)$ is for pulsatile flows. based deconvolution method can be used to determine the relative volumetric flow for both constant flows and pulsatile flows.

One important conclusion that can be drawn from this paper is that the pulsation effects can be ignored for flow analysis if only relative flow analysis is required. It was also shown that the proposed deconvolution method is effective even on real measurement data. With such a technique, applications of the RLS-based method include the removal of effects of noninstantaneous injections and the alleviation of imaging problems caused by limited acoustic access.

The simulation results indicate the good agreement, under constant flows, between the MTT values and the washout time constants (i.e., $V / Q$ ). However, for pulsatile flow results under the same conditions, the MTTs were consistently larger than the washout time constants. In other words, the mean flow rate of a pulsatile flow may not adequately represent the rate of dilution of the microbubbles. In addition, the measured time constants were consistently smaller than the theoretical values. Thus, it is possible that the effective mixing volume is smaller than the physical volume of the compartment. In other words, although good correlation between the measurements and the theory has been established in this paper under various flow conditions, it must be emphasized that only relative flow estimation can be achieved. Various issues, including the two observations described previously, must be understood before quantitative flow analysis is possible using TIC-based methods.

Acknowledgements-Support from the National Health Research Institutes of R.O.C. (grant no. NHRI-EX91-9127EI) is greatly appreciated. The authors also thank the reviewers for insightful comments.

\section{REFERENCES}

Bates JHT. Deconvolution of tracer and dilution data using the Wiener filter. IEEE Trans Biomed Eng 1991;38:1262-1266.

Burns PN. Harmonic imaging with ultrasound contrast agents. Clin Radiol 1996;51(Suppl. 1):50-55.

Cheirif B, Willian AZ, Roberto B, et al. Assessment of regional myocardial perfusion by contrast echocardiography. II Detection of changes in transmural and subendocardial perfusion during dipyridamole-induced hyperemia in a model of critical coronary stenosis. J Am Coll Cardiol 1989;14:1555-1565.

Chen X, Schwarz KQ, Phillips D, Steinmetz SD, Schlief R. A mathematical model for the assessment of hemodynamic parameters using quantitative contrast echocardiography. IEEE Trans Biomed Eng 1998;45:754-765.

Claasen TACM, Mecklenbrauker WFG. On stationary linear timevarying systems. IEEE Trans Circuits Syst 1982;29:169-184.

de Jong N. Improvements in ultrasound contrast agents. IEEE Eng Med Biol 1996;15:72-82.

DiBella EVR, Gullberg GT, Ross SG, Christian PE. Compartmental modeling of ${ }^{18}$ FDG in the heart using dynamic SPECT. Nuclear Sci Sympos 1997;2:1460-1463.

Gobbel GT, Fike JR. A deconvolution method for evaluating indicatordilution curves. Phys Med Biol 1994;39:1833-1854.

Goldberg BB. Ultrasound contrast agents. London: Dunitz, 1997, 1-8. 
Gramiak R, Shah PM, Kramer DH. Ultrasound cardiography: contrast studies in anatomy and function. Radiology 1969;92:939-948.

Haider B, Chiao RY. Higher order nonlinear ultrasonic imaging. IEEE Ultrason Sympos 1999;2:1527-1531.

Haykin S. Adaptive filter theory. London: Prentice Hall International, 1991.

Heikkonen J, Mantyla M. Application of compartmental analysis in liver blood flow measurements using Xe-133. Eng Med Biol Soc 1991;13:313-314.

Hoff L. Nonlinear response of sonazoid. numerical simulation about pulse-inversion and subharmonics. IEEE Ultrason Sympos 2000;2: 1885-1888.

Kaul S, Kelly P, Oliner JD, et al. Assessment of regional myocardial blood flow with myocardial contrast two-dimensional echocardiography. J Am Coll Cardiol 1989;13:468-482.

Kemper AJ, Force T, Kloner R, et al. Contrast echocardiographic estimation of regional myocardial blood flow after acute coronary occlusion. Circulation 1985;72:1115-1124.

Kirkhorn J, Frinking PJA, de Jong N, Torp H. Three-stage approach to ultrasound contrast detection. IEEE Trans Ultrason Ferroelec Freq Control 2001;48:1013-1022.

Krishnan S, Hamilton JD, O'Donnell M. Suppression of propagating second harmonic in ultrasound contrast imaging. IEEE Trans Ultrason Ferroelec Freq Control 1998;45:704-711.

Li P-C, Yeh C-K, Wang S-W. Time-intensity-based volumetric flow measurement: An in vitro study. Ultrasound Med Biol 2002;28: 349-358.

Ljung L, Soderstrom T. Theory and practice of recursive identification. Cambridge, MA: MIT Press, 1983.

Meikle SR, Eberl S, Fulton RR, Kassiou M. The influence of tomograph sensitivity on parameter estimation in small animal imaging studies. Nuclear Sci Sympos 1998;1898-1903.

Morgan K, Averkiou M, Ferrara K. The effect of the phase of transmission on contrast agent echoes. IEEE Trans Ultrason Ferroelec Freq Control 1998;45:872-875.

Nouri-Baranger T, Piquer E, Canet E. A new coronary model for MRI perfusion studies. 2001 Proceedings of the 23rd Annual International Conference of the IEEE. Eng Med Biol Soc 2001;1:109-112.

Ophir J, Parker KJ. Contrast agents in diagnostic ultrasound. Ultrasound Med Biol 1989;15:319-333.

Ostrander LE, Lee BY, Silverman DG, Groskopf RW. A compartment model for fluorometric indication of tissue perfusion. IEEE Trans Biomed Eng 1990;37:1017-1023.
Phillips PJ. Contrast pulse sequences: Imaging nonlinear microbubble. IEEE Ultrason Sympos 2001;2:1739-1745.

Porter TR, D'Sa A, Turner C, et al. Myocardial contrast echocardiography for the assessment of coronary blood flow reserve: Validation in humans. J Am Coll Cardiol 1993;21:349-355.

Schwarz KQ, Bezante GP, Chen X, Mottley JG, Schlief R. Volumetric arterial flow quantification using echo contrast. An in vitro comparison of three ultrasonic intensity methods: radio frequency, video and Doppler. Ultrasound Med Biol 1993a;19:447-460.

Schwarz KQ, Bezante GP, Chen X, Schlief R. Quantitative echo contrast concentration measurement by Doppler sonography. Ultrasound Med Biol 1993b;19:289-297.

Schwarz KQ, Chen X, Bezante GP, Phillips D, Schlief R. The Doppler kinetics of microbubble echo contrast. Ultrasound Med Biol 1996; 22:453-462.

Schoning M, Scheel, P. Color duplex measurement of cerebral blood flow volume: Infra- and interobserver reproducibility and habituation to serial measurements in normal subjects. J Cereb Blood Flow Metab 1996;16:523-31.

Seidel G, Meyer K, Metzler V, et al. Human cerebral perfusion analysis with ultrasound contrast agent constant infusion: A pilot study on healthy volunteers. Ultrasound Med Biol 2002;28:183-189.

Shi WT, Forsberg F, Raichlen JS, Needleman L, Goldberg BB. Pressure dependence of subharmonic signals from contrast microbubbles. Ultrasound Med Biol 1999;25:275-283.

Shung KK, Wilson BP, Flenniken RR. Cardiovascular applications of ultrasonic contrast media. IEEE Ultrason Sympos 1992;2:11551158.

Wilson B, Shung KK, Hete B, Levene H, Barnhart JL. A feasibility study on quantitating myocardial perfusion with Albunex ${ }^{\circledR}$, an ultrasonic contrast agent. Ultrasound Med Biol 1993;19:181-191.

Yeh C-K, Wang S-W, Li P-C. Feasibility study of time-intensity-based blood flow measurements using deconvolution. Ultrason Imaging 2001;23:90-105.

Yeh C-K, Yang M-J, Li P-C. Contrast-specific ultrasonic flow measurements based on both input and output time-intensities. Ultrasound Med Biol 2003;29:671-678.

Zhou YA, Huang SC, Cloughesy T, Hoh CK, Black K. A modelingbased factor extraction method for determining spatial heterogeneity of Ga-68 EDTA kinetics in brain tumor. IEEE Trans Nuclear Sci 1997;44:2522-2526. 